\title{
ChemComm
}

\section{Boronic acid ester with dopamine as a tool for bioconjugation and for visualization of cell apoptosis $\dagger$}

\author{
Cite this: Chem. Commun., 2014 \\ 50,6390 \\ Received 29th November 2013, \\ Accepted 14th April 2014
}

DOI: $10.1039 / c 3 c c 49100 e$

www.rsc.org/chemcomm

\author{
Wei Scarano, Hongxu Lu and Martina H. Stenzel*
}

\begin{abstract}
Boronic esters are investigated as a pathway to conjugate biological molecules to polymers. Although the stability of the boronic ester was slightly dependent on the molecular weight of the polymer and the $\mathrm{pH}$ value, the conjugates were stable when micelles were endocytosed by cells. Only the onset of apoptosis led to cleavage of the boronic ester.
\end{abstract}

The conjugation of proteins, peptides or other biomolecules to polymers has been an active research field for many years now. The high activity in this field is evidenced by the large number of review articles. ${ }^{1-4}$ The common feature of these reactions is the formation of permanent linkers although disulfides, which are the result of the reaction between pyridyl disulfide (PDS) and thiols, can be cleaved under reductive conditions. An alternative pathway could be the reaction between boronic acids and alcohols. Boronic acids can undergo reactions with a range of compounds, most notably with 1,2-dihydroxyl functionalities, with high affinity via reversible boronate esters formation. (Scheme 1). ${ }^{5}$ It is possibly one of the strongest single-pair reversible functional group interactions in an aqueous environment ${ }^{6}$ and it has already been utilized for various biomedical applications. ${ }^{6-8}$ Sumerlin and co-workers demonstrated recently that boronic acids are robust during radical polymerization ${ }^{9}$ allowing the design of polymers for glucose sensing. ${ }^{10}$ These encouraging results inspired us to test this chemistry for conjugation of biomolecules to the surface of micelles. Particularly appealing is the potentially reversible nature of the boronic acid ester as a tool to detect $\mathrm{pH}$ changes. Tumour tissue, which is transiently or chronically hypoxic, has a $\mathrm{pH}$ slightly more acidic than the blood and normal tissues, with a mean $\mathrm{pH}$ of 6.5-7.0. In addition the endocytic pathway of cells begins near the physiological $\mathrm{pH}$ of 7.4 , it drops to a lower $\mathrm{pH}$ of 5.5-6.0 in endosomes and approaches $\mathrm{pH}$

Centre for Advanced Macromolecular Design, School of Chemistry,

University of New South Wales, Sydney, New South Wales, Australia.

E-mail: M.Stenzel@unsw.edu.au

$\dagger$ Electronic supplementary information (ESI) available: Synthesis and experimental part, details on equilibrium constant determination, ${ }^{11} \mathrm{~B}$ NMR, fluorescent confocal microscopy of cells incubated with albendazole loaded micelles and of cells with empty micelle in the presence of $\mathrm{Ca}^{2+}$ ions. See DOI: 10.1039/c3cc49100e

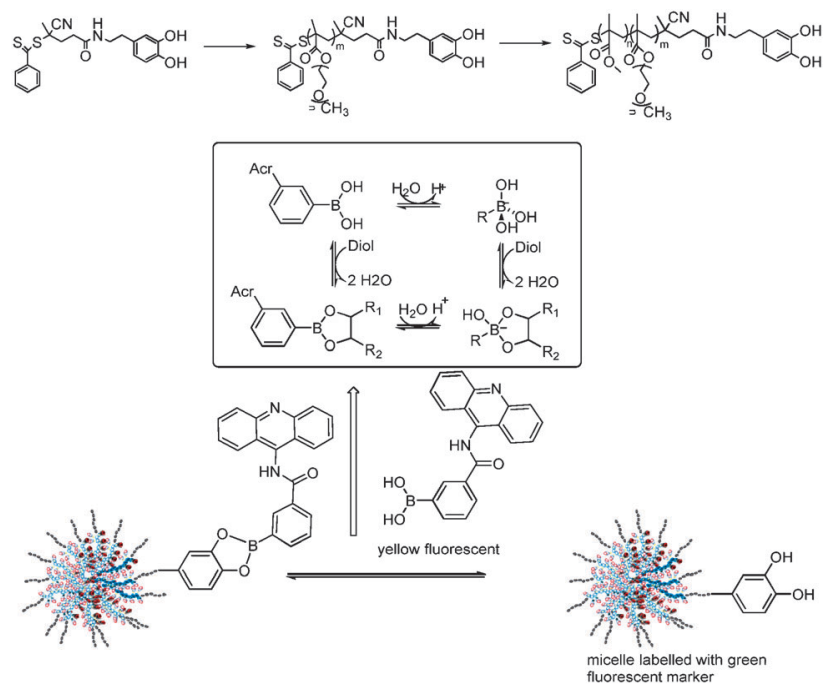

Scheme 1 Micelles conjugated to 9-amino acridine via boronic acid ester and the relationship between phenylboronic acid and its diol ester. ${ }^{13}$

4.5-5.0 in lysosomes. ${ }^{11}$ Aim is to explore boronic esters as an efficient tool for bioconjugation. The focus of this work will be the stability of the boronic ester in in vitro studies using healthy cells and cells that undergo apoptosis. We therefore developed a RAFT (reversible addition fragmentation chain transfer) agent ${ }^{12}$ based on dopamine, which is known to have a high affinity towards boronic acid (Scheme 1). Subsequent polymerization leads either to watersoluble POEGMEMA polymers or, after chain extension with MMA, to micelles, which can readily react with boronic acid. The use of a boronic acid derivative, functionalized with the yellow fluorescent 9-amino acridine, allows direct monitoring of the complex in the cell.

Micelles, which can conjugated to biomolecules via boronic acid esters need to be based on block copolymer with an end functionality that is known to react with boronic acid in an efficient manner such as 1,2-dihydroxy benzene (catechol). A catechol-terminated RAFT agent (Fig. S1, ESI $\dagger$ ) was employed in the polymerization of oligo (ethylene glycol) methyl ether methacrylate (OEGMEMA) (Table 1, B1 and B2) and the chain extension with methyl 
Table 1 Summary of SEC measured molecular weights of homopolymers and block copolymers, polydispersity index $\Theta$ and the hydrodynamic diameter $D_{\mathrm{h}}$ of the micelles

\begin{tabular}{llrrl}
\hline & & $\begin{array}{l}\mathrm{Mn}^{\mathrm{SEC}} \\
\left(\mathrm{gmol}^{-1}\right)\end{array}$ & \multicolumn{1}{c}{$\mathrm{D}$} & \multicolumn{1}{l}{$\begin{array}{l}D_{\mathrm{h}} \text { in water/ } \\
\mathrm{nm}(\mathrm{PDI})\end{array}$} \\
\hline B1 & POEGMEMA $_{15}$ & 4800 & 1.19 & - \\
B2 & POEGMEMA $_{32}$ & 11000 & 1.26 & - \\
B3 & POEGMEMA $_{32}-\mathrm{MMA}_{71}$ & 21700 & 1.20 & $93(0.191)$ \\
B4 & POEGMEMA(F) $_{28}$ & 9800 & 1.24 & \\
B5 & POEGMEMA(F) $)_{28}-b-(t \mathrm{BMA})_{175}$ & 21800 & 1.46 & $42(0.126)$ \\
\hline
\end{tabular}

methacrylate (MMA) to obtain the block copolymer POEGMEMA ${ }_{32}-$ block-PMMA ${ }_{71}$ (B3). ${ }^{1} \mathrm{H}$ NMR shows confirms high endgroup fidelity (Fig. S2, ESI $†$ ). Two other polymers $\mathbf{B} 4$ and $\mathbf{B 5}$ were synthesized in similar fashion to incorporate fluorescein for the tagging of polymer which can be observed in confocal microscope studies. The purpose of polymer $\mathbf{B} \mathbf{5}$ was to create a carrier for platinum drugs to study the stability of the bioconjugation during apoptosis. The amphiphilic block copolymer B3 was self-assembled by adding water slowly to a solution of the polymer in DMF. Dialysis against water to remove DMF leads to micelles with a hydrodynamic diameter of $50 \mathrm{~nm}$ according to dynamic light scattering (DLS) analysis (Table 1, Fig. S3, ESI $\dagger$ ). In addition $\mathbf{B 5}$ were loaded with toxic anticancer agents albendazole $(\mathrm{ABZ})^{14}$ and platinum drugs ${ }^{15}$ to monitor the behaviour of the boronic ester under stress.

Prerequisite to understand the nature of the boronic ester bond at different $\mathrm{pH}$ values and also the effect of the length and type of polymer is the determination of the equilibrium constant. Fluorescence spectroscopy was used to study the binding between the polymers listed in Table 1 and boronic acid. The determination of the binding constant was carried out by using competitive assay containing the catechol dye Alizarin Red S (ARS), boronic acid and a secondary catechol of choice. ${ }^{16}$ In the presence of boronic acid or boronate functional groups the catechol portion of the ARS forms a boronate ester which emits fluorescence upon excitation. The formation of this boronate ester can be observed as fluorescence intensity increase with the addition of boronic acid to an ARS solution until equilibrium is reached (Fig. S4, ESI $\dagger$ ). The binding constant between ARS and boronic acid $\left(K_{\text {eq }}\right)$ can be calculated using Benesi-Hildebrand method (see ESI $\dagger)^{17}$ and the values were listed in Table 2. The calculations were based on the equilibrium constant between ARS and PBA and the competition between ARS and B1-B3 for the boronic ester (Scheme 2).

The values listed in Table 2 highlight the lower stability of the boronic acid ester in an acidic environment. Considering the equilibrium depicted in Scheme 1 this is not unexpected. Moreover, the values reveal the dependency on the molecular weight. The binding constants of dopamine terminated polymers are slightly reduced compared to dopamine itself. In addition, the calculated

Table 2 Association constant $K_{\text {eq } 1}$ of ARS-PBA complex and association constant $K_{\text {eq }}$ of the diol-PBA complex $\left(\mathrm{M}^{-1}\right)$

\begin{tabular}{lrllll}
\hline & ARS & Dopamine & B1 & B2 & B3 Micelle \\
\hline pH 7 & 1443 & 448 & 315 & 275 & 376 \\
pH 5 & 650 & 415 & 216 & 185 & 256
\end{tabular}

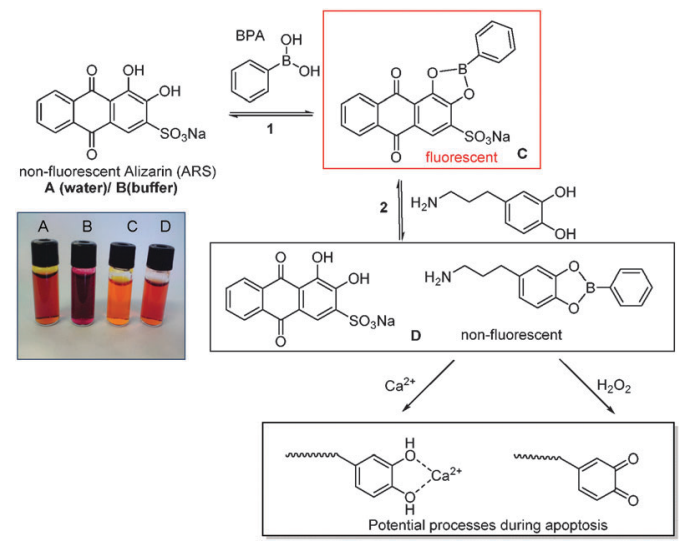

Scheme 2 Determination of association constant $K_{\text {eq }}$ between boronic acid and dopamine and the potential cleavage of the boronic ester during apoptosis.

binding constant decreases with increasing molecular weight of the polymer. The trend of reduced binding efficiency with increasing molecular weight could potentially be caused by steric hindrance and lower accessibility of the dopamine functionality. Interestingly, the calculated binding constant of the micelle is higher than the POEGMEMA polymers alone. This can possibly be understood considering the altered structure of the polymer chains. The conformation of POEGMEMA is more likely to take on a stretched chain conformation similar to a brush-like structure exposing the dopamine functionalities to the surface of the micelle. However, the origin of this effect is unclear and should be investigated in more detail.

The pH-responsive nature was also confirmed using ${ }^{11} \mathrm{~B}$-NMR spectroscopy. ${ }^{18} \mathrm{PBA}$ is predominately in the $\mathrm{sp}^{2}$-hybridised form as evidenced by the chemical shift of $29.6 \mathrm{ppm}$. Upon addition of dopamine the ${ }^{11} \mathrm{~B}$ signal shifted to $9.24 \mathrm{ppm}$ indicating the formation of the tetrahedral boronate ester species. The experiment was repeated adjusting the $\mathrm{pH}$ value to 5 , which liberates the free PBA (Fig. S6, ESI $\dagger$ ).

According to these spectroscopic studies above the boronic ester should respond to $\mathrm{pH}$ changes in the cell such as the ones found in the endosomes and lysosomes. To test the suitability of boronic esters for bioconjugation, an acridine based fluorescent derivative was synthesized (Scheme 1). Micelles with dopamine functionalities on the surface (and labelled with green fluorescent marker) were then mixed with the acridine boronic acid dye (yellow fluorescent). From the previous fluorescence studies with PBA it is known that the conjugation is almost instantaneous. The central question is if the conjugation can survive the acidic endocytic pathway once these micelles have been internalized by cancer cell lines, here human ovarian carcinoma Ovcar-3. Confocal fluorescent microscopy allows the direct observation of the localization of the green and the yellow fluorescent dyes. The micelles employed were based on POEGMEMA as the hydrophilic block and $t$-butyl acrylate ( $t$ BMA) as the hydrophobic block (B5). The co-localization of the yellow acridine phenyl boronic acid compound, the green fluorescent micelles and the lysosymes stained with red LysoTracker DND-99 indicates the presence of 


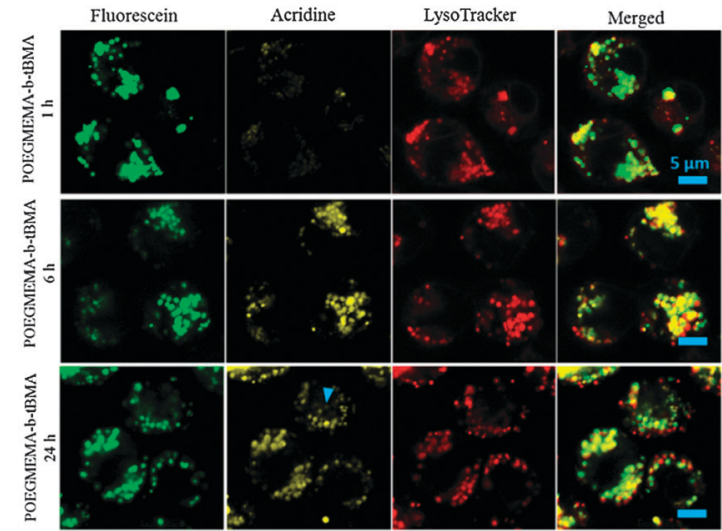

Fig. 1 Confocal microphotographs of Ovcar-3 cells after incubation with POEGMEMA $26-b-t B A_{175}$ micelles at $37{ }^{\circ} \mathrm{C}$ for $1 \mathrm{~h}, 6 \mathrm{~h}$ and $24 \mathrm{~h}$; the polymer (green) was labeled with fluorescein. Acridine (yellow) was added to the micelles. Lysosomes (red) were stained with LysoTracker Red DND-99. Blue triangles indicate the intracellular parts with only acridine but without fluorescein-labeled polymers. Scale bar $=5 \mu \mathrm{m}$.

the micelles in the lysosomes which confirms the expected endocytic pathway (Fig. 1). The co-localization of the green and yellow fluorescent dye can be observed up to 24 hours. After that, the acridine is visible in places where micelles are not (blue arrow in Fig. 1). The conjugation seems stable for an extended period of time. Boronic esters can therefore be considered as a fast and efficient way of conjugation.

Next, micelles were loaded with oxoplatin, a highly efficient anti-cancer drug. The synthesis of these micelles and their toxicity are described elsewhere. ${ }^{15}$ Upon uptake of the drug loaded micelles, cell apoptosis occurs resulting in the detachment of the acridine derivative from the micelle (Fig. 2). Over the next 24 hours, more and more cells die while simultaneously acridine can be found distributed all over the cytosol. Another system was tested to confirm the observation. This time the anti-cancer drug albendazole (ABZ) was physically encapsulated into the same micelle. Again, upon cell death the acridine detaches from the micelles (Fig. S7, ESI $\dagger$ ). The split between acridine and micelle with $\mathrm{ABZ}$ is less dramatic and highlights that the observed effect is subject to toxicity of the drug used and the amount of drug loaded. More detailed studies on these two parameters are therefore warranted. It is unlikely that the slightly lower $\mathrm{pH}$ value of the cytosol during apoptosis (around $\mathrm{pH} 7.0 \mathrm{vs}$. 7.4, drop of 0.3-0.4 $\mathrm{pH}$ units $^{19}$ ) could cause the cleavage of the boronic ester since the ester was obviously stable while residing in the lysosomes and endosomes. Also changes of the micelle itself such as disassociation are unlikely since it was not observed with the empty micelle.

These observations during apoptosis cannot be explained without turning to cell biology. Apoptosis causes an increase in caspase activity, which results in the cleavage of more than 100 different proteins. ${ }^{20} \mathrm{~A}$ further event is the increase in the level of peroxides during apoptosis ${ }^{21}$ and can potentially lead to the oxidation of dopamine including $o$-quinone and other structures. $^{22}$ Recently, boronic acid in combination with a FRET pair has been used as a sensor for the formation of peroxides

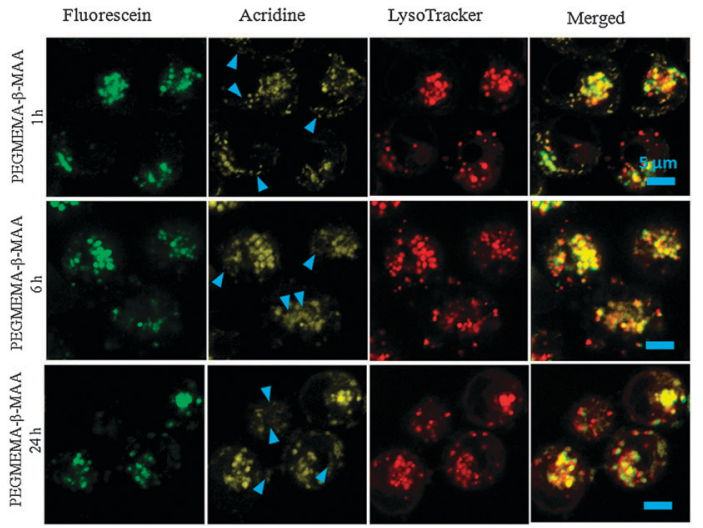

Fig. 2 Confocal microphotographs of Ovcar-3 cells after incubation with toxic POEGMEMA $26-b-M_{17 A_{17}}$ Pt micelles at $37{ }^{\circ} \mathrm{C}$ for $1 \mathrm{~h}, 6 \mathrm{~h}$ and $24 \mathrm{~h}$. Polymers (green) were labeled with fluorescein. Acridine (yellow) was added to the micelles. Lysosomes (red) were stained with LysoTracker Red DND-99. Blue triangles indicate the intracellular parts with only acridine but without fluorescein-labeled polymers. Scale bar $=5 \mu \mathrm{m}$.

inside cells. $^{23}$ The driving force in the reported case was the oxidation of boronic acid to $p$-quinone methide (and loss of the boronic acid). ${ }^{23,24}$ However, incubation of the boronic ester used here with $\mathrm{H}_{2} \mathrm{O}_{2}$ resulted in the formation of the original boronic acid due to the difference in structure, as evidenced by ${ }^{11} \mathrm{~B}$ NMR (Fig. S9, ESI $\dagger$ ), which probably coincided with the oxidation of dopamine to $o$-quinone, thus, this reaction could be one of the culprits that led to the split of acridine from the micelle. In addition, elevated $\mathrm{Ca}^{2+}$ ion levels are measured during apoptosis. ${ }^{25}$ The $\mathrm{Ca}^{2+}$ ions are efficient signalling agents in the body, which can readily form complexes with 1,2-dihydroxy benzene (catechol) derivatives such as dopamine. To test this hypothesis, the fluorescent binding assay was carried out in the presence of $\mathrm{Ca}^{2+}$ (Fig. S8, ESI $\dagger$ ). The strong fluorescence between ARS and PBA was reduced when adding $\mathrm{Ca}^{2+}$ since the bivalent ion could bind strongly to ARS. Addition of dopamine to the ARS/PBA/Ca ${ }^{2+}$ results in increased fluorescence since dopamine can liberate ARS from $\mathrm{Ca}^{2+}$. To further elucidate the effect observed in Fig. 2, the experiment in Fig. 1 with the non-toxic micelles was repeated, but with additionally added $\mathrm{Ca}^{2+}$ ions. The concentration of $\mathrm{Ca}^{2+}$ ions $(8.4 \mathrm{mM})$ was chosen at a suitable range to avoid toxic effects to the cells. In contrast to Fig. 1, where the cleavage of the yellow acridine from the green fluorescent micelles was absent, the presence of $\mathrm{Ca}^{2+}$ ions caused an effect similar to the one observed when cytotoxic drugs were added (Fig. S10, ESI $\dagger$ ).

While it is very difficult to clearly isolate the origin of this effect, it seems that the formation of peroxides and $\mathrm{Ca}^{2+}$ ions may contribute to the boronic ester cleavage, but it is crucial to consider that other parameters may also play a role.

In conclusion, conjugation via boronic esters presents an efficient pathway to attach biomolecules to polymers. Although the complex is pH-responsive, it is reasonably stable under physiological condition. However, the susceptibility of the catechol functionality to oxidation and complex formation result in the disassociation of the boronic complex during apoptosis where increased oxidation 
and $\mathrm{Ca}^{2+}$ influx could potentially lead to the liberation of the boronic acid, thus it can be used to monitor the onset of apoptosis.

\section{Notes and references}

1 G. N. Grover and H. D. Maynard, Curr. Opin. Chem. Biol., 2010, 14, 818-827.

2 A. K. Shakya, H. Sami, A. Srivastava and A. Kumar, Prog. Polym. Sci., 2010, 35, 459-486.

3 B. Le Droumaguet and J. Nicolas, Polym. Chem., 2010, 1, 563-598.

4 M. A. Gauthier and H.-A. Klok, Polym. Chem., 2010, 1, 1352-1373.

5 H. Ito, Y. Kono, A. Machida, Y. Mitsumoto, K. Omori, N. Nakamura, Y. Kondo and K. Ishihara, Inorg. Chim. Acta, 2003, 344, 28-36.

6 J. N. Cambre and B. S. Sumerlin, Polymer, 2011, 52, 4631-4643.

7 M. L. Stolowitz, C. Ahlem, K. A. Hughes, R. J. Kaiser, E. A. Kesicki, G. S. Li, K. P. Lund, S. M. Torkelson and J. P. Wiley, Bioconjugate Chem., 2001, 12, 229-239.

8 S. Moffatt, C. Papasakelariou, S. Wiehle and R. Cristiano, Gene Ther., 2006, 13, 761-772.

9 P. De, S. R. Gondi, D. Roy and B. S. Sumerlin, Macromolecules, 2009, 42, 5614-5621.

10 D. Roy, J. N. Cambre and B. S. Sumerlin, Chem. Commun., 2009, 2106-2108.
11 N. Murthy, J. Campbell, N. Fausto, A. S. Hoffman and P. S. Stayton, J. Controlled Release, 2003, 89, 365-374.

12 A. Gregory and M. H. Stenzel, Prog. Polym. Sci., 2012, 37, 38-105.

13 G. Springsteen and B. H. Wang, Tetrahedron, 2002, 58, 5291-5300.

14 Y. Kim, M. H. Pourgholami, D. L. Morris and M. H. Stenzel, Macromol. Biosci., 2011, 11, 219-233.

15 W. Scarano, H. T. T. Duong, H. Lu, P. L. De Souza and M. H. Stenzel, Biomacromolecules, 2013, 14, 962-975.

16 J. Yan, G. Springsteen, S. Deeter and B. H. Wang, Tetrahedron, 2004, 60, 11205-11209.

17 H. R. Mulla, N. J. Agard and A. Basu, Bioorg. Med. Chem. Lett., 2004, 14, 25-27.

18 D. Reed, eMagRes, John Wiley \& Sons, Ltd, 2007.

19 S. Matsuyama, J. Llopis, Q. L. Deveraux, R. Y. Tsien and J. C. Reed, Nat. Cell Biol., 2000, 2, 318-325.

20 C. Köhler, S. Orrenius and B. Zhivotovsky, J. Immunol. Methods, 2002, 265, 97-110.

21 A. Gorman, A. McGowan and T. G. Cotter, FEBS Lett., 1997, 404, 27-33.

22 J. Segura-Aguilar, D. Metodiewa and C. J. Welch, Biochim. Biophys. Acta, 1998, 1381, 1-6.

23 R. Weinstain, E. N. Savariar, C. N. Felsen and R. Y. Tsien, J. Am. Chem. Soc., 2013, 136, 874-877.

24 G. C. Van de Bittner, E. A. Dubikovskaya, C. R. Bertozzi and C. J. Chang, Proc. Natl. Acad. Sci. U. S. A., 2010, 107, 21316-21321.

25 M. J. Berridge, M. D. Bootman and P. Lipp, Nature, 1998, 395, 645-648. 